SUBJECT AREAS:

TWO-DIMENSIONAL

MATERIALS

ELECTRONIC STRUCTURE

Received

11 September 2013

Accepted

29 November 2013

Published

16 December 2013

Correspondence and requests for materials should be addressed to X.D.P. (xdpi@zju.edu. $\mathrm{cn})$ or D.R.Y.

(mseyang@zju.edu.cn)

\section{Silicene oxides: formation, structures and electronic properties}

\author{
Rong Wang', Xiaodong $\mathrm{Pi}^{1}$, Zhenyi $\mathrm{Ni}^{1}$, Yong Liu', Shisheng Lin², Mingsheng X $\mathrm{u}^{3}$ \& Deren Yang ${ }^{1}$
}

'State Key Laboratory of Silicon Materials and Department of Materials Science and Engineering, Zhejiang University, Hangzhou 310027, China, ${ }^{2}$ State Key Laboratory of Modern Optical Instrumentation and Department of Information Science and Electronic Engineering, Zhejiang University, Hangzhou 310027, China, ${ }^{3}$ Department of Polymer Science and Engineering, Zhejiang University, Hangzhou 310027 , China.

Understanding the oxidation of silicon has been critical to the success of all types of silicon materials, which are the cornerstones of modern silicon technologies. For the recent experimentally obtained two-dimensional silicene, oxidation should also be addressed to enable the development of silicene-based devices. Here we focus on silicene oxides (SOs) that result from the partial or full oxidation of silicene in the framework of density functional theory. It is found that the formation of SOs greatly depends on oxidation conditions, which concern the oxidizing agents of oxygen and hydroxyl. The honeycomb lattice of silicene may be preserved, distorted or destroyed after oxidation. The charge state of $\mathrm{Si}$ in partially oxidized silicene ranges from +1 to +3 , while that in fully oxidized silicene is +4 . Metals, semimetals, semiconductors and insulators can all be found among the SOs, which show a wide spectrum of electronic structures. Our work indicates that the oxidation of silicene should be exquisitely controlled to obtain specific SOs with desired electronic properties.

T he progress of the experimental synthesis of silicene, the silicon counterpart of graphene, has been generating great interest ${ }^{1-8}$. It is conjectured that silicene is even more advantageous than graphene ${ }^{9-14}$. For instance, the spin-orbit interaction of silicene is stronger than that of graphene, leading to detectable quantum spinHall effects in silicene ${ }^{14,15}$. The salient structural feature of buckling for silicene facilitates the tuning of the electronic properties of silicene by external fields ${ }^{16-18}$. In addition, silicene is clearly much more compatible with the existing well-established silicon technologies than graphene. Silicene-based devices may be readily incorporated into current silicon integrated circuits.

When silicene-based devices are fabricated, the oxidation of silicene is of the great question during the processing ${ }^{19,20}$. This is due to the fact that silicon routinely reacts with oxygen to form silicon oxide, which has been playing a critical role in the success of all types of current silicon materials such as bulk silicon ${ }^{21}$, silicon nanowires $^{22,23}$ and silicon nanocrystals ${ }^{24,25}$. It is rather reasonable that the oxidation of silicene should also be addressed to realize the full potential of silicene in all kinds of applications. De Padova et al. ${ }^{19}$ and Molle et al. ${ }^{20}$ have recently shown that the oxidation of silicene only starts at a high dose $(1000 \mathrm{~L})$ of pure molecular oxygen. The relatively low reactivity of silicene to molecular oxygen may be due to the added $\mathrm{sp}^{2}$ hybridization of silicon atoms ${ }^{19}$. When exposed to air, however, silicene is readily oxidized without identified reasons ${ }^{20}$. This indicates that the mechanism for the oxidation of silicene may seriously depend on oxidation conditions.

Up to now, very limited work has been carried out to investigate silicene oxides (SOs) that result from the oxidation of silicene, although it is imperative that the properties of SOs should be elucidated to guide the design of silicene-based device structures. By means of first-principles calculation, Wang et al. ${ }^{26}$ have recently studied the mechanical and electronic properties of a specific SO with the stoichiometry of Si:O = 1: 1 (silicene monoxide). The silicene monoxide is obtained by analogizing its formation to that of a thermal-reduction-induced graphene monoxide ${ }^{27}$. In fact, the graphene monoxide can only be produced by the thermal reduction of a multilayer graphene oxide. Diffusion in the multilayer structure limits the reduction process, leading to the kinetics-controlled formation of graphene monoxide ${ }^{27}$. It is clear that the current non-existence of multilayer silicene oxides disables the formation of silicene monoxide in a fashion similar to graphene monoxide. In the present work, we begin with silicene, which is composed of a single layer of silicon atoms. Oxidizing agents such as atomic oxygen $(\mathrm{O})$ and hydroxyl $(\mathrm{OH})$ in a variety of bonding configurations are incorporated into the lattice of silicene to form SOs. The formation of all the SOs is evaluated in the point of view of thermodynamics. It turns out that the charge state of $\mathrm{Si}$ in partially oxidized silicene ranges from +1 to +3 , while that of $\mathrm{Si}$ in fully oxidized silicene is +4 . When silicene is oxidized by $\mathrm{O}$, single-side over-bridging $\mathrm{O}\left(\mathrm{O}_{o b}^{s}\right)$ is the most likely incorporated $\mathrm{O}$ configuration 
during the oxidation. If $\mathrm{OH}$ is the oxidizing agent, boat-like $\mathrm{OH}$ $\left(\mathrm{OH}_{b l}\right)$ and umbrella-like $\mathrm{OH}\left(\mathrm{OH}_{u l}\right)$ may be the most likely incorporated in the resulting SOs. When $\mathrm{O}$ and $\mathrm{OH}$ both exist, fully oxidized silicene may be readily produced. The structural characteristics of all the SOs have been identified in this work. We find that the electronic properties of SOs significantly depend on the bonding of $\mathrm{O}$ and $\mathrm{OH}$. Metallic, semimetallic, semiconducting and insulating SOs can all be obtained.

\section{Results}

Partially oxidized silicene. Figure 1 shows the optimized structures of silicene and SOs that result from the partial oxidation of silicene with both top and side views. The characteristics of all the optimized structures are tabulated in Table 1 (notations of all the $\mathrm{O}$ and $\mathrm{OH}$ configurations are introduced in Table S1 in the Supporting Information). In the single-atom-thick honeycomb lattice of silicene, the bond length of Si-Si $(2.28 \AA)$, the bond angle of Si-Si$\mathrm{Si}\left(116^{\circ}\right)$ and the buckling distance $(0.45 \AA)$ are all consistent with those reported in previous works ${ }^{15,28-30}$ (Figure 1 (a)). When each Si$\mathrm{Si}$ bond is transformed to a $\mathrm{Si}-\mathrm{O}-\mathrm{Si}$ bond by an $\mathrm{O}$ atom during the oxidation of silicene, the buckling of $\mathrm{Si}$ atoms actually disappears in the resulting $\mathrm{SO}$ (Figure 1 (b)). All the $\mathrm{O}$ atoms are also in the same
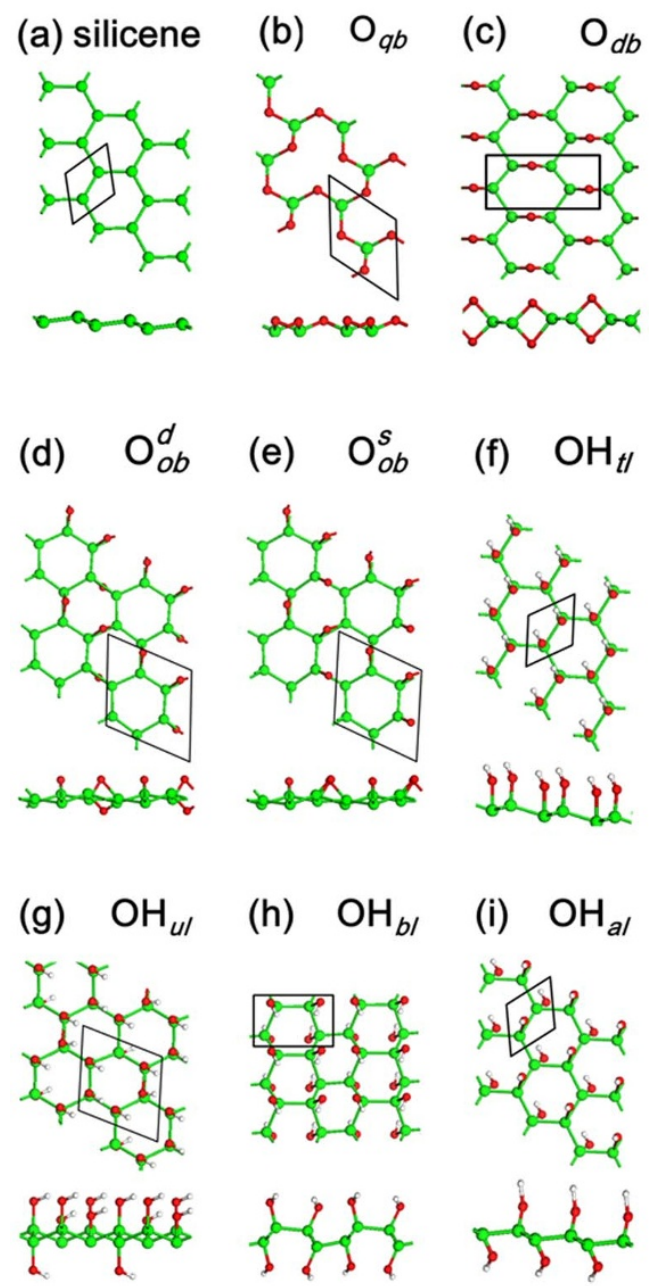

- $\mathrm{Si}$
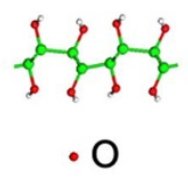

plane, which is only $0.02 \AA$ away from that of $\mathrm{Si}$ atoms. Therefore, quasi-in-plane bridging $\mathrm{O}\left(\mathrm{O}_{q b}\right)$ can be used to denote the incorporated $\mathrm{O}$. It is likely that each $\mathrm{Si}$-Si bond at only one pair of opposite sides in a hexagonal ring is oxidized. But the oxidation results in two $\mathrm{Si}-\mathrm{O}-\mathrm{Si}$ bonds, which are symmetric with respect to the oxidation-flatted plane of Si atoms (Figure 1 (c)). This leads to the configuration of double bridging $\mathrm{O}\left(\mathrm{O}_{d b}\right)$ for the incorporated $\mathrm{O}$. An $\mathrm{O}$ atom may also overbridge two neighboring $\mathrm{Si}$ atoms via their unpaired electrons. The overbridging $\mathrm{O}$ atoms can be located at either both sides $\left(\mathrm{O}_{o b}^{d}\right)$ or single side $\left(\mathrm{O}_{o b}^{s}\right)$ of the original silicene (Figure 1 (d) and Figure 1 (e)). When the oxidizing agent is $\mathrm{OH}$, each $\mathrm{Si}$ atom may be simply bonded to an $\mathrm{OH}$ group via the unpaired electron of Si. For a single hexagonal ring in the honeycomb lattice of silicene, the number of $\mathrm{OH}$ groups located at one side of the hexagonal ring may be zero, one, two or three. We denote the $\mathrm{OH}$ groups in the resulting SOs as top-like $\mathrm{OH}\left(\mathrm{OH}_{t l}\right)$ (Figure $1(\mathrm{f})$ ), umbrella-like $\mathrm{OH}\left(\mathrm{OH}_{u l}\right)$ (Figure $1(\mathrm{~g})$ ), boat-like $\mathrm{OH}\left(\mathrm{OH}_{b l}\right)$ (Figure $1(\mathrm{~h})$ ) and armchair-like $\mathrm{OH}\left(\mathrm{OH}_{a l}\right)$ (Figure 1 (i)), respectively. Please note that $\mathrm{OH}_{a l}$ and $\mathrm{OH}_{b l}$ alternately passivate $\mathrm{Si}$ atoms with a step of one and two $\mathrm{Si}$ atoms at both sides of the original silicene, respectively. Among all the oxidizing agents only $\mathrm{OH}_{a l}$ and $\mathrm{OH}_{t l}$ do not change the original arrangement of $\mathrm{Si}$ atoms in silicene. The buckling distance, $\mathrm{Si}-\mathrm{Si}$ bond length and $\mathrm{Si}-\mathrm{Si}$-Si bond angel of the $\mathrm{SO}$ with $\mathrm{OH}_{a l}$ or $\mathrm{OH}_{t l}$ are all the same as those of silicene (Table 1). In the lattices of the SOs with $\mathrm{OH}_{b l}$ and $\mathrm{OH}_{u l}$ all the Si-Si-Si bond angles decrease to be between 103 and $113^{\circ}$. The Si-Si bonds in the $\mathrm{SO}$ with $\mathrm{OH}_{b l}$ are either stretched or compressed, while those in the SO with $\mathrm{OH}_{u l}$ are all stretched. For the SO with $\mathrm{O}_{d b}$ the bond length of $\mathrm{Si}-\mathrm{Si}$ and bond angle of $\mathrm{Si}$-Si-Si decrease to $2.19 \AA$ and $106^{\circ}$, respectively. All the $\mathrm{Si}-\mathrm{Si}$ bonds are stretched and some of $\mathrm{Si}-\mathrm{Si}-\mathrm{Si}$ bond angles increase up to $122^{\circ}$ in the $\mathrm{SO}$ with $\mathrm{O}_{o b}^{s}$ or $\mathrm{O}_{o b}^{d}$. All the above-mentioned structural changes lead to the distortion of the honeycomb lattice of silicene. The buckling distances of $\mathrm{Si}$ atoms in the distorted honeycomb lattices of the SOs with $\mathrm{OH}_{b l}, \mathrm{OH}_{u l}$, $\mathrm{O}_{d b}, \mathrm{O}_{o b}^{s}$ and $\mathrm{O}_{o b}^{d}$ are 1.14, 0.03, 0, 0.02 and $0.03 \AA$, respectively. It is clear that no $\mathrm{Si}-\mathrm{Si}$ bonds exist in the $\mathrm{SO}$ with $\mathrm{O}_{q b}$ (Figure 1 (b)). The length of $\sim 83 \% \mathrm{Si}-\mathrm{O}$ bonds is $1.91 \AA$, while that of the remaining $\mathrm{Si}$ O bonds is $1.42 \AA$. The Si-O-Si bond angles fall in the range from 104 to $112^{\circ}$. All the structural characteristics of the $\mathrm{SO}$ with $\mathrm{O}_{q b}$ indicate that the honeycomb lattice of silicene is destroyed by the incorporation of $\mathrm{O}_{q b}$.

We have worked out the formula of each SO by examining its unit cell (Table 1). The formulas of the SOs with $\mathrm{O}_{q b}, \mathrm{O}_{d b}, \mathrm{O}_{o b}^{a}, \mathrm{O}_{o b}^{s}, \mathrm{OH}_{t l}$, $\mathrm{OH}_{u l}, \mathrm{OH}_{b l}$ and $\mathrm{OH}_{a l}$ are $\mathrm{Si}_{2} \mathrm{O}_{3}, \mathrm{Si}_{4} \mathrm{O}_{4}, \mathrm{Si}_{6} \mathrm{O}_{3}, \mathrm{Si}_{6} \mathrm{O}_{3}, \mathrm{Si}_{2}(\mathrm{OH})_{2}$, $\mathrm{Si}_{6}(\mathrm{OH})_{6}, \mathrm{Si}_{4}(\mathrm{OH})_{4}$ and $\mathrm{Si}_{2}(\mathrm{OH})_{2}$, respectively. Therefore, the charge states of $\mathrm{Si}$ are +3 and +2 for the SOs with $\mathrm{O}_{q b}$ and $\mathrm{O}_{d b}$, respectively. The charge states of $\mathrm{Si}$ are all +1 for the SOs with $\mathrm{O}_{o b}^{a}, \mathrm{O}_{o b}^{s}, \mathrm{OH}_{t l}$, $\mathrm{OH}_{u l}, \mathrm{OH}_{b l}$ and $\mathrm{OH}_{a l}$. It is clear that the highest charge state $(+4)$ of $\mathrm{Si}$ has not been obtained in these SOs. Therefore, they only result from the partial oxidation of silicene.

The formation energy $\left(\mathrm{E}_{f}\right)$ per atom of oxygen in a SO can be calculated by using

$$
E_{f}=\left(E_{t}-E_{s}-\frac{N_{O}}{2} \mu_{O_{2}}-N_{O H} \mu_{O H}\right) / N_{O}
$$

where $\mathrm{E}_{t}$ is the total energy of the SO. $\mathrm{E}_{s}$ is the total energy of silicene. $N_{\mathrm{O}}$ and $N_{\mathrm{OH}}$ are the numbers of $\mathrm{O}$ and $\mathrm{OH}$, respectively. $\mu_{\mathrm{O}_{2}}$ and $\mu_{\mathrm{OH}}$ are the chemical potentials of $\mathrm{O}_{2}$ and $\mathrm{OH}$, respectively. Figure 2 shows the change of $\mathrm{E}_{f}$ with respect to $\Delta \mu_{\mathrm{OH}}$ for each SO by fixing at the total energy of oxygen gas $(-7.5 \mathrm{eV}) . \Delta \mu_{\mathrm{OH}}$ is the difference between $\mu_{\mathrm{OH}}$ in the oxidation system of silicene and that in water $(-10.8 \mathrm{eV})$. For the SOs induced by the partial oxidation with $\mathrm{O}$ (e. g., in the atmosphere of oxygen gas), $\mathrm{E}_{f}$ increases in the order of $\mathrm{O}_{o b}^{d}$, $\mathrm{O}_{o b}^{d}, \mathrm{O}_{d b}$ and $\mathrm{O}_{q b}$. For those induced by the partial oxidation with $\mathrm{OH}$ (e. g., in water), $\mathrm{E}_{f}$ increases in the order of $\mathrm{OH}_{b l} / \mathrm{OH}_{u l}, \mathrm{OH}_{a l}$ and

Figure 1 Optimized structures of (a) silicene and silicene oxides with (b) $\mathrm{O}_{q b}$, (c) $\mathrm{O}_{d b}$, (d) $\mathrm{O}_{o b}^{d}$, (e) $\mathrm{O}_{o b}^{s}$, (f) $\mathrm{OH}_{t b}$, (g) $\mathrm{OH}_{u b}$ (h) $\mathrm{OH}_{b l}$ and (i) $\mathrm{OH}_{a l}$. Both top and side views of the structures are shown. The unit cell of each structure is indicated by a rhombus or rectangle. $\mathrm{Si}, \mathrm{O}$ and $\mathrm{H}$ atoms are denoted by green, red and grey balls, respectively. 
Table 1 Formulas and structural properties such as the buckling distance $(\Delta)$ of $\mathrm{Si}$, the bond length of $\mathrm{Si}-\mathrm{Si}$ and the bond angle of $\mathrm{Si}$ $\mathrm{Si}$-Si for silicene and partially oxidized silicene $\left(\mathrm{SO}\right.$ s with $\mathrm{O}_{q b}, \mathrm{O}_{d b}$, $\mathrm{O}_{o b}^{d}, \mathrm{O}_{o b}^{s} \mathrm{OH}_{t,}, \mathrm{OH}_{u l}, \mathrm{OH}_{b l}$ and $\left.\mathrm{OH}_{a l}\right)$. Each bandgap $\left(E_{g}\right)$ is obtained with the PBE correlation exchange functional. The more accurate values of $E_{g}$ obtained with the B3LYP correlation exchange functional are shown in the brackets

\begin{tabular}{lllccc} 
& Formula & $\Delta(\AA)$ & $\langle\mathrm{Si}-\mathrm{Si}>(\AA)$ & $\angle(\mathrm{Si}-\mathrm{Si}-\mathrm{Si})\left({ }^{\circ}\right)$ & $E_{g}(\mathrm{eV})$ \\
\hline Silicene & $\mathrm{Si}_{2}$ & 0.45 & 2.28 & 116 & $0(0)$ \\
$\mathrm{O}_{q b}$ & $\mathrm{Si}_{2} \mathrm{O}_{3}$ & 0 & --- & --- & $5.2(6.6)$ \\
$\mathrm{O}_{d b}$ & $\mathrm{Si}_{4} \mathrm{O}_{4}$ & 0 & 2.19 & 106 &.-- \\
$\mathrm{O}_{o b}^{d}$ & $\mathrm{Si}_{6} \mathrm{O}_{3}$ & 0.03 & 2.34 & $110-122$ & $0.5(0.7)$ \\
$\mathrm{O}_{o b}^{s}$ & $\mathrm{Si}_{6} \mathrm{O}_{3}$ & 0.02 & 2.34 & $110-122$ & $0.3(0.6)$ \\
$\mathrm{OH}_{t l}$ & $\mathrm{Si}_{2}(\mathrm{OH})_{2}$ & 0.45 & 2.28 & 116 &.-- \\
$\mathrm{OH}_{b l}$ & $\mathrm{Si}_{6}(\mathrm{OH})_{6}$ & 0.03 & 2.34 & $104-113$ & $0.2(0.4)$ \\
$\mathrm{OH}_{b l}$ & $\mathrm{Si}_{4}(\mathrm{OH})_{4}$ & 1.14 & $2.06-2.34$ & $103-112$ & $0(0)$ \\
$\mathrm{OH}_{a l}$ & $\mathrm{Si}_{2}(\mathrm{OH})_{2}$ & 0.45 & 2.28 & 116 & $0.4(0.4)$
\end{tabular}

$\mathrm{OH}_{t l}$. Therefore, we should expect that the oxidation of silicene in the atmosphere of oxygen gas the most likely leads to the formation of the $\mathrm{SO}$ with $\mathrm{O}_{o b}^{s}$. When silicene is only oxidized by $\mathrm{OH}$, the SOs with $\mathrm{OH}_{b l}$ and $\mathrm{OH}_{u l}$ are the most likely produced.

We now move to the electronic properties of all the partially oxidized silicene. Figure 3 shows the band structure and density of states (DOS) of each partially oxidized silicene. For comparison, the band structure and DOS of silicene have also been included in Figure 3. Consistent with previous results, silicene is semimetallic with a zero bandgap $^{11,14,26}$ (Figure 3 (a)). The top of the valence band and the bottom of the conduction band linearly cross at the Fermi level, where the DOS is negligible. $\mathrm{O}_{q b}$ opens the bandgap of silicene by

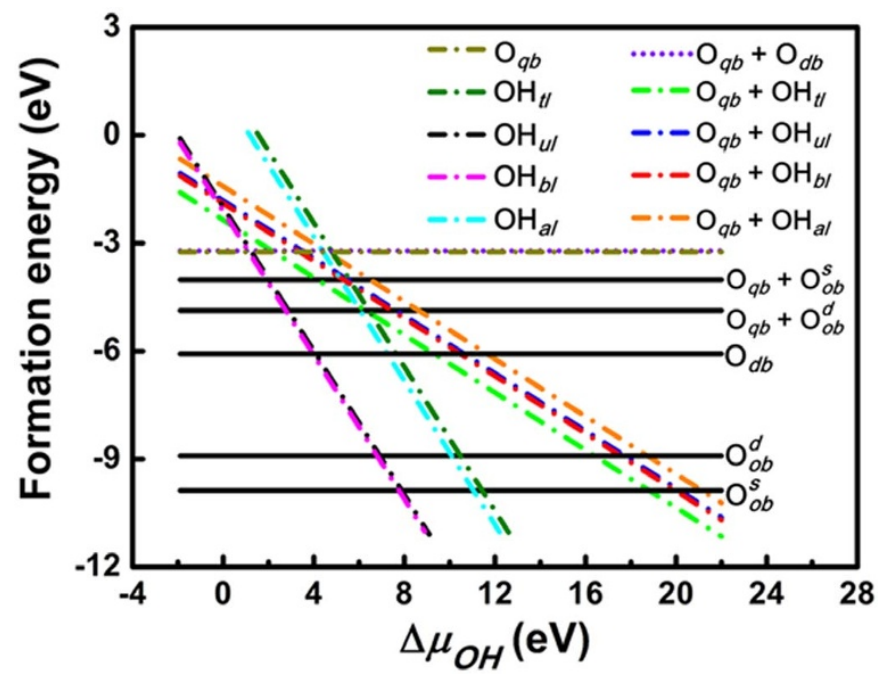

Figure $2 \mid$ Formation energy of a silicene oxide with $\mathrm{O}_{q b}, \mathrm{O}_{d b}, \mathbf{O}_{o b}^{d}, \mathbf{O}_{o b}^{s}$, $\mathrm{OH}_{t b}, \mathrm{OH}_{u b}, \mathrm{OH}_{b l}, \mathrm{OH}_{a b}, \mathrm{O}_{q b}+\mathrm{O}_{d b}, \mathrm{O}_{q b}+\mathrm{O}_{o b}^{s}, \mathrm{O}_{q b}+\mathrm{O}_{o b}^{d}, \mathrm{O}_{q b}+\mathrm{OH}_{t b}$, $\mathrm{O}_{q b}+\mathrm{OH}_{u l}, \mathrm{O}_{q b}+\mathrm{OH}_{b l}$ or $\mathrm{O}_{q b}+\mathrm{OH}_{a l}$ with respect to the difference between the chemical potential of $\mathrm{OH}$ in the oxidation system of silicene and that in water.

$5.2 \mathrm{eV}$ (Figure 3 (b)). Such a large bandgap means that the SO with $\mathrm{O}_{q b}$ is actually an insulator. We notice that there are two deep energy levels in the bandgap of the $\mathrm{SO}$ with $\mathrm{O}_{q b}$. By examining the partial DOS of the $\mathrm{SO}$ with $\mathrm{O}_{q b}$ (Figure $\mathrm{S} 1$ in the Supporting Information), we find that the two deep energy levels mainly originate from the $\mathrm{p}$ orbital of $\mathrm{O}_{q b}$. Both the p-orbital of $\mathrm{Si}$ and the p-orbital of $\mathrm{O}$ significantly contribute to the conduction band of the $\mathrm{SO}$ with $\mathrm{O}_{q b}$. The
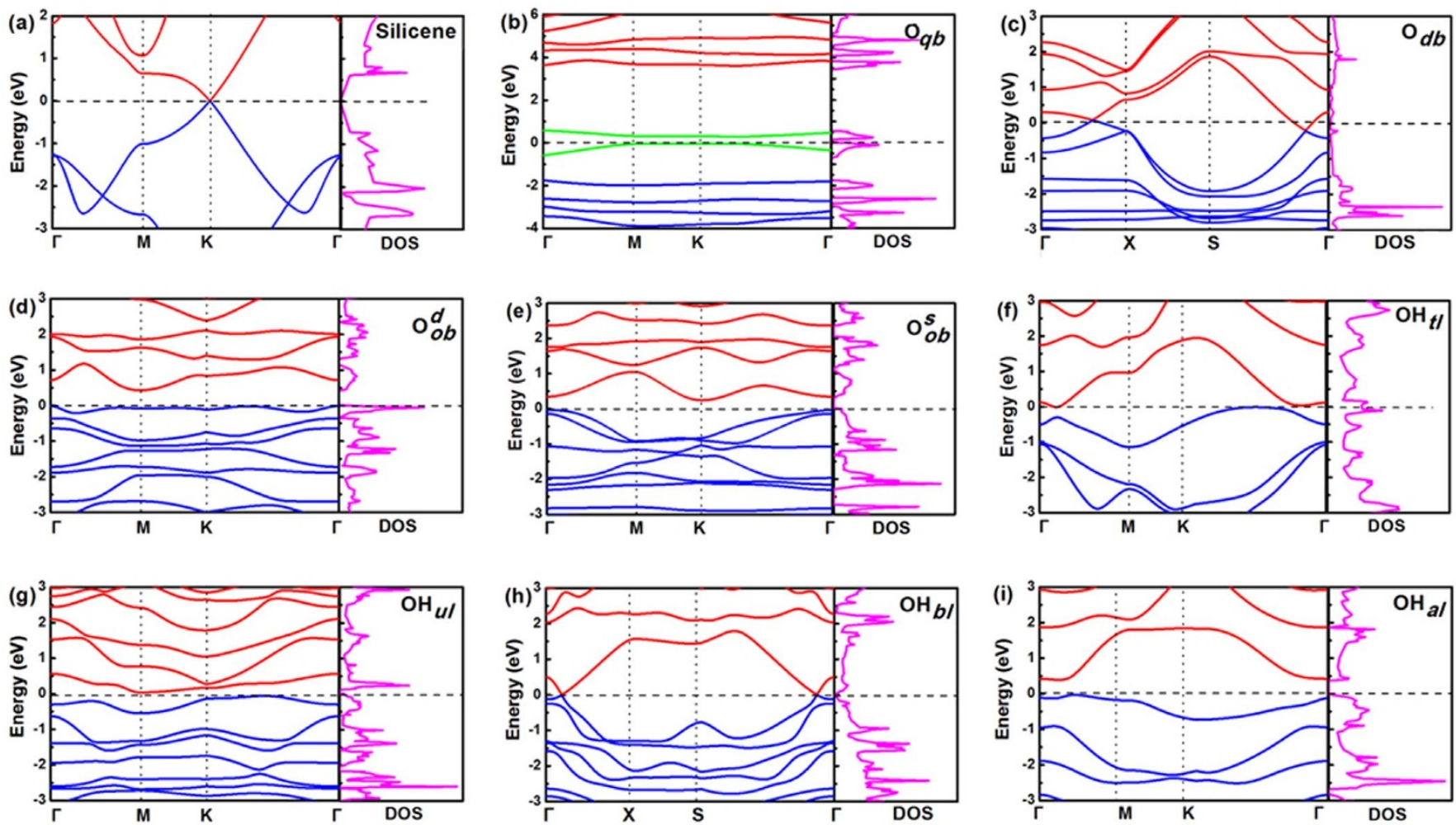

Figure $3 \mid$ Band structures and density of states (DOS) of (a) silicene and a silicene oxide with (b) $\mathrm{O}_{q b}$, (c) $\mathrm{O}_{d b}$, (d) $\mathrm{O}_{o b}^{d}$, (e) $\mathrm{O}_{o b}^{s}$, (f) $\mathrm{OH}_{t b},(\mathrm{~g}) \mathrm{OH}_{u l}$, (h) $\mathrm{OH}_{b l}$ or (i) $\mathrm{OH}_{a l} . \Gamma, \mathrm{K}, \mathrm{M}$ are special points in the first Brillouin zone of a cubic crystal. $\Gamma, \mathrm{X}, \mathrm{S}$ are special points in the first Brillouin zone of a hexagonal crystal. Energy is shifted so that the Fermi level is $0 \mathrm{eV}$ (the horizontal dashed line). Conduction bands, valance bands, deep energy levels and DOS are indicated by red, blue, green and pink lines, respectively. 
valence band of the $\mathrm{SO}$ with $\mathrm{O}_{q b}$ mainly originates from the p-orbital of $\mathrm{O}$. It is known that a bandgap is usually underestimated in the density functional theory (DFT) based on the Perdew-BurkeErnzerhof (PBE) correlation exchange functional. Thus, we have also performed DFT calculations based on the Becke, three-parameter, Lee-Yang-Parr (B3LYP) exchange-correlation functional to get the more accurate bandgap. For the $\mathrm{SO}$ with $\mathrm{O}_{q b}$, the B3LYP bandgap is $6.6 \mathrm{eV}$ (Table 1).

The Fermi level is located in the conduction band for the $\mathrm{SO}$ with $\mathrm{O}_{d b}$ (Figure $3(\mathrm{c})$ ), indicating that the $\mathrm{SO}$ with $\mathrm{O}_{d b}$ is a metal. Figure 3 (d) shows that the $\mathrm{SO}$ with $\mathrm{O}_{o b}^{d}$ is an indirect-bandgap semiconductor. The valence-band maximum appears at $\Gamma$ point, while the conduction-band minimum appears at $\mathrm{M}$ point. The bandgap is as large as $0.5 \mathrm{eV}$ (the B3LYP bandgap is $0.7 \mathrm{eV}$ ). The $\mathrm{SO}$ with $\mathrm{O}_{o b}^{s}$ is also an indirect-bandgap semiconductor (Figure 3 (e)). The valenceband maximum and conduction-band minimum are located at $\Gamma$ point and $\mathrm{K}$ point, respectively. The bandgap is $0.3 \mathrm{eV}$ (the B3LYP bandgap is $0.6 \mathrm{eV}$ ). There are no energy levels in the bandgap of the $\mathrm{SO}$ with $\mathrm{O}_{o b}^{d}$ or $\mathrm{O}_{o b}^{s}$. The origins of the conduction band and valence band for the $\mathrm{SO}$ with $\mathrm{O}_{d b}, \mathrm{O}_{o b}^{d}$ or $\mathrm{O}_{o b}^{s}$ are basically similar to those for the $\mathrm{SO}$ with $\mathrm{O}_{q b}$ (Figure $\mathrm{S} 1$ in the Supporting Information).

For the $\mathrm{SO}$ with $\mathrm{OH}_{t l}$ the Fermi level is located at the bottom of the conduction band (Figure 3 (f)). The DOS near the Fermi level is relatively large. Therefore, the $\mathrm{SO}$ with $\mathrm{OH}_{t l}$ is actually a metal. For the $\mathrm{SO}$ with $\mathrm{OH}_{u l}$, the valence-band maximum and conduction-band minimum are located at $\mathrm{K}^{\prime}$ point (between $\mathrm{K}$ point and $\Gamma$ point) and $\mathrm{M}$ point, respectively (Figure $3(\mathrm{~g})$ ). The bandgap is $0.2 \mathrm{eV}$ (the B3LYP bandgap is $0.3 \mathrm{eV}$ ). As shown in Figure $3(\mathrm{~h}$ ), the Fermi level crosses both the valence-band maximum and conduction-band minimum for the SO with $\mathrm{OH}_{b l}$. The DOS at the Fermi level is negligible. Clearly, the $\mathrm{SO}$ with $\mathrm{OH}_{b l}$ is also a semimetal. The valence-band maximum and the conduction-band minimum of the SO with $\mathrm{OH}_{a l}$ are located at $\mathrm{M}^{\prime}$ point (between $\Gamma$ point and $\mathrm{M}$ point) and $\Gamma^{\prime}$ point (between $\Gamma$ point and M point), respectively (Figure $3(\mathrm{i})$ ). The indirect bandgap of the $\mathrm{SO}$ with $\mathrm{OH}_{a l}$ is $0.4 \mathrm{eV}$ (the B3LYP bandgap is also $0.4 \mathrm{eV}$ ). The valence band of the SO with one type of all the $\mathrm{OH}$ configurations also mainly originates from the p-orbital of $\mathrm{O}$. The s-orbital of $\mathrm{H}$ now significantly contributes to the conduction band together with the p-orbital of $\mathrm{O}$ and the p-orbital of $\mathrm{Si}$ (Figure S1 in the Supporting Information).

For all the semiconducting partially oxidized silicene (SOs with $\mathrm{O}_{o b}^{d}, \mathrm{O}_{o b}^{s}, \mathrm{OH}_{u l}$ and $\mathrm{OH}_{a l}$ ), we have calculated the effective masses $\left(\mathrm{m}^{*}\right)$ of electrons and holes by using the method adopted by Ni et al. ${ }^{17}$ and Li et al. ${ }^{31}$ (Table S2 in the Supporting Information). It is found that $\mathrm{m}^{*}$ is between 0.36 and $4.78 \mathrm{~m}_{0}\left(\mathrm{~m}_{0}\right.$ is the electron rest mass). According to $\mu=e \tau / m^{*}$ ( $\mu$ is the carrier mobility, $\mathrm{e}$ is the charge of an electron, $\tau$ is the scattering time that is assumed to approximate to that in graphene), we obtain that the carrier mobilities of the semiconducting partially oxidized silicene are in the order of magnitude of $10-10^{2} \mathrm{~cm}^{2} \mathrm{~V}^{-1} \mathrm{~s}^{-1}$ (Table S2 in the Supporting Information).

Fully oxidized silicene. We find that $\mathrm{O}_{q b}$ should be incorporated together with other types of $\mathrm{O}$ or $\mathrm{OH}$ configurations to achieve the charge state of +4 for $\mathrm{Si}$ in fully oxidized silicene. Figure 4 shows the SOs based on the combination of $\mathrm{O}_{q b}$ and one of the configurations of $\mathrm{O}_{d b}, \mathrm{O}_{o b}^{d}, \mathrm{O}_{o b}^{s}, \mathrm{OH}_{t l}, \mathrm{OH}_{u l}, \mathrm{OH}_{b l}$ and $\mathrm{OH}_{a l}$. Please note that the number of $\mathrm{O}_{q b}$ atoms is reduced by one third for the $\mathrm{SO}$ with $\mathrm{O}_{q b}+$ $\mathrm{O}_{d b}$ because the existence of $\mathrm{O}_{d b}$ only allows four $\mathrm{O}_{q b}$ atoms to be incorporated in a hexagonal ring. The buckling distances of $\mathrm{Si}$ atoms in the SO with $\mathrm{O}_{q b}+\mathrm{O}_{d b}$ and $\mathrm{O}_{q b}+\mathrm{O}_{o b}^{d}$ are 0.04 and $0.06 \AA$, respectively. Si atoms in all the other SOs are located in the same plane. This is the same as what happens to the $\mathrm{SO}$ only with $\mathrm{O}_{q b}$. By examining the unit cells (Figure 4), we obtain that the formulas of the SOs with $\mathrm{O}_{q b}+\mathrm{O}_{d b}, \mathrm{O}_{q b}+\mathrm{O}_{o b}^{d}, \mathrm{O}_{q b}+\mathrm{O}_{o b}^{s}, \mathrm{O}_{q b}+\mathrm{OH}_{t l}, \mathrm{O}_{q b}+\mathrm{OH}_{u l}$, $\mathrm{O}_{q b}+\mathrm{OH}_{b l}$ and $\mathrm{O}_{q b}+\mathrm{OH}_{a l}$ are $\mathrm{Si}_{2} \mathrm{O}_{4}, \mathrm{Si}_{6} \mathrm{O}_{12}, \mathrm{Si}_{6} \mathrm{O}_{12}, \mathrm{Si}_{4} \mathrm{O}_{6}(\mathrm{OH})_{4}$, (a) $\mathrm{O}_{q b}+\mathrm{O}_{d b}$

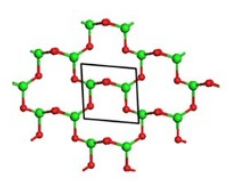

(b) $\mathrm{O}_{q b}+\mathrm{O}_{o b}^{d}$

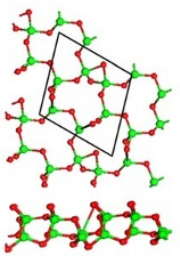

(c) $\mathrm{O}_{q b}+\mathrm{O}_{o b}^{s}$

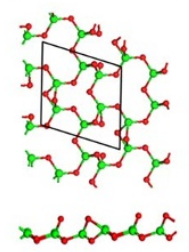

(d) $\mathrm{O}_{q b}+\mathrm{OH}_{t /}$

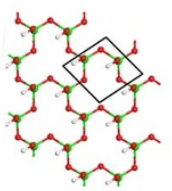

insing. (e) $\mathrm{O}_{q b}+\mathrm{OH}_{u l}$

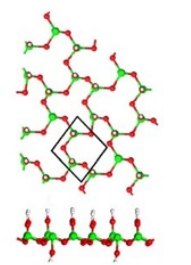

(f) $\mathrm{O}_{q b}+\mathrm{OH}_{b l}$
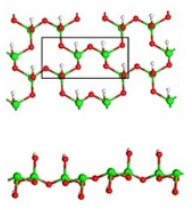

(g) $\mathrm{O}_{q b}+\mathrm{OH}_{a l}$
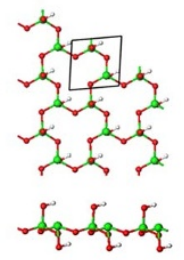

Figure 4 Optimized structures of silicene oxides with (a) $\mathrm{O}_{q b}+\mathrm{O}_{d b}$, (b) $\mathrm{O}_{q b}+\mathrm{O}_{o b}^{d}$, (c) $\mathrm{O}_{q b}+\mathrm{O}_{o b}^{s}$, (d) $\mathrm{O}_{q b}+\mathrm{OH}_{t b}$ (e) $\mathrm{O}_{q b}+\mathrm{OH}_{u b},(\mathrm{f}) \mathrm{O}_{q b}+\mathrm{OH}_{b l}$ and (g) $\mathrm{O}_{q b}+\mathrm{OH}_{a l}$. Both top and side views are shown. The unit cell of each structure is indicated by a rhombus or rectangle. $\mathrm{Si}, \mathrm{O}$ and $\mathrm{H}$ atoms are denoted by green, red and grey balls, respectively.

$\mathrm{Si}_{6} \mathrm{O}_{9}(\mathrm{OH})_{6}, \mathrm{Si}_{2} \mathrm{O}_{3}(\mathrm{OH})_{2}$ and $\mathrm{Si}_{2} \mathrm{O}_{3}(\mathrm{OH})_{2}$, respectively (Table $\mathrm{S} 3$ in the Supporting Information).

The formation energy of each fully oxidized silicene is also shown in Figure 2. When silicene is only oxidized in the atmosphere of oxygen gas, the most likely formed fully oxidized silicene is the SO with $\mathrm{O}_{q b}+\mathrm{O}_{o b}^{d}$. If silicene is oxidized in an environment in which both $\mathrm{O}$ and $\mathrm{OH}$ exist, a high chemical potential of $\mathrm{OH}$ may most likely lead to the formation of the $\mathrm{SO}$ with $\mathrm{O}_{q b}+\mathrm{OH}_{t l}$.

Figure 5 shows the band structure and DOS of each fully oxidized silicene. For the $\mathrm{SO}$ with $\mathrm{O}_{q b}+\mathrm{OH}_{u l}$ the energy levels introduced by $\mathrm{OH}_{u l}$ can basically fill up the bandgap that is opened by $\mathrm{O}_{q b}$. Therefore, the full oxidation of silicene with $\mathrm{O}_{q b}+\mathrm{OH}_{u l}$ gives rise to a metallic product. The PBE (B3LYP) bandgaps of the SOs with $\mathrm{O}_{q b}+\mathrm{O}_{d b}, \mathrm{O}_{q b}+\mathrm{OH}_{b l}$ and $\mathrm{O}_{q b}+\mathrm{OH}_{a l}$ are 5.1 (6.4), 5.0 (5.4) and 5.1 (7.2) eV, respectively. This indicates that the SOs with $\mathrm{O}_{q b}+\mathrm{O}_{d b}$, $\mathrm{O}_{q b}+\mathrm{OH}_{b l}$ and $\mathrm{O}_{q b}+\mathrm{OH}_{a l}$ are insulators. Nevertheless, we should notice that there are deep energy levels in the rather wide bandgaps of these three insulators (Figure 5 (a), (f) and (g)). The SOs with $\mathrm{O}_{q b}+$ $\mathrm{O}_{o b}^{d}, \mathrm{O}_{q b}+\mathrm{O}_{o b}^{s}$ and $\mathrm{O}_{q b}+\mathrm{OH}_{t l}$ are all indirect-bandgap semiconductors. Their PBE (B3LYP) bandgaps are 2.8 (3.9), 3.2 (4.3) and $2.1(3.6) \mathrm{eV}$, respectively. Again, we see deep energy levels in the bandgaps of these three semiconductors. It should be pointed out that both the valance bands and conduction bands of all the fully oxidized silicene mainly originate from the p-orbital of O (Figure S1 in the Supporting Information). This signifies that O plays the dominant role for the electronic properties of all the fully oxidized silicene. The effective masses of electrons and holes for the semiconducting fully oxidized silicene are between 0.73 and 87.81 $\mathrm{m}_{0}$, leading to carrier mobilities in the order of magnitude of a few to $10^{2} \mathrm{~cm}^{2} \mathrm{~V}^{-1} \mathrm{~s}^{-1}$ (Table $\mathrm{S} 2$ in the Supporting Information).

\section{Discussion}

De Padova et al. ${ }^{19}$ and Molle et al..$^{20}$ have recently carried out experiments on the oxidation of silicene in the atmosphere of oxygen gas. The low charge state $(+1$ and +2$)$ of Si observed after the high oxygen exposure $(1000 \mathrm{~L})$ of silicene may be attributed to the formation of the SOs with $\mathrm{O}_{o b}^{s}, \mathrm{O}_{o b}^{d}$ and $\mathrm{O}_{d b}$, which have lower $\mathrm{E}_{f}$. The $\mathrm{E}_{f}$ of the $\mathrm{SO}$ with $\mathrm{O}_{q b}$ is even higher than those of the SOs with $\mathrm{O}_{q b}+\mathrm{O}_{o b}^{s}$ and $\mathrm{O}_{q b}+\mathrm{O}_{o b}^{d}$, and comparable to that of the $\mathrm{SO}$ with $\mathrm{O}_{q b}+\mathrm{O}_{d b}$. This means that +3 cannot be the dominant charge state for $\mathrm{Si}$ in 

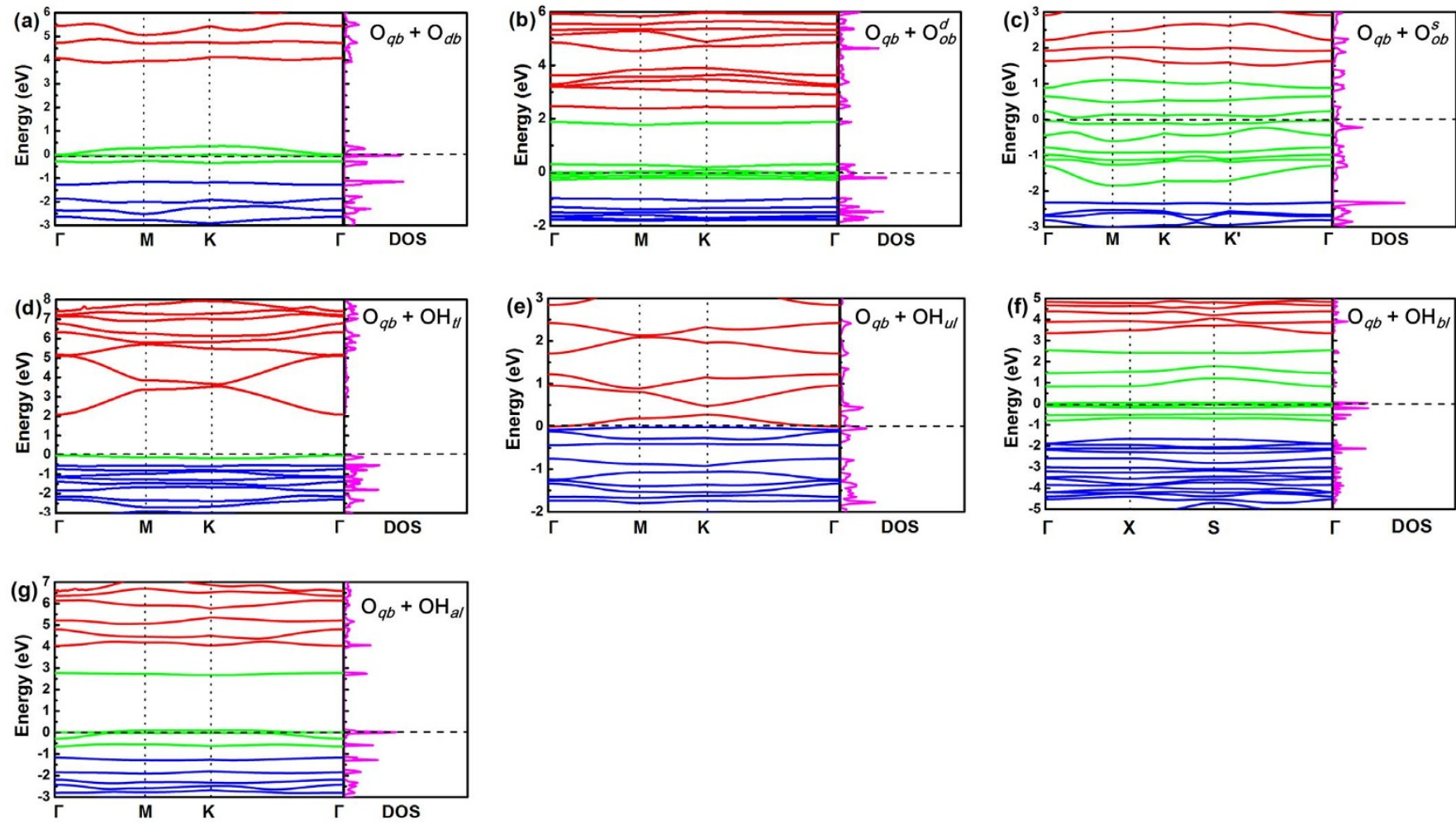

Figure $5 \mid$ Band structure and density of states (DOS) of a silicene oxide with (a) $\mathrm{O}_{q b}+\mathrm{O}_{d b}$, (b) $\mathrm{O}_{q b}+\mathrm{O}_{o b}^{d}$, (c) $\mathrm{O}_{q b}+\mathrm{O}_{o b}^{s}$, (d) $\mathrm{O}_{q b}+\mathrm{OH}_{t b}$, (e) $\mathrm{O}_{q b}+$ $\mathrm{OH}_{u b}$, (f) $\mathrm{O}_{q b}+\mathrm{OH}_{b l}$ or $(\mathrm{g}) \mathrm{O}_{q b}+\mathrm{OH}_{a l} . \Gamma, \mathrm{K}, \mathrm{K}^{\prime}, \mathrm{M}$ are special points in the first Brillouin zone of a cubic crystal. $\Gamma, \mathrm{X}, \mathrm{S}$ are special points in the first Brillouin zone of a hexagonal crystal. Energy is shifted so that the Fermi level is $0 \mathrm{eV}$ (horizontal dashed line). Conduction bands, valance bands, deep energy levels and DOS are indicated by red, blue, green and pink lines, respectively.

only O-oxidized silicene. The charge state of Si may directly change from $+1 /+2$ to +4 with the advancement of silicene oxidation in the atmosphere of oxygen gas ${ }^{19}$. Please note that silicene cannot be fully oxidized if it is only oxidized by $\mathrm{OH}$ (Figure 2 and Table 1). The charge state of $\mathrm{Si}$ in the final $\mathrm{OH}$-induced $\mathrm{SO}$ is always +1 unless $\mathrm{H}$ is detached from $\mathrm{OH}$ to give rise to restructuring.

When silicene is oxidized in air, $\mathrm{O}$ from oxygen molecules and $\mathrm{OH}$ from water may both account for the oxidation. It appears that in Molle et al.'s work ${ }^{20}$ the air was an $\mathrm{OH}$-rich environment, which lowered the $\mathrm{E}_{f}$ of each $\mathrm{OH}$-incorporated $\mathrm{SO}$. The oxidation of silicene was easily initiated to produce the SOs with all types of $\mathrm{OH}$ configurations, in which the charge state of $\mathrm{Si}$ was all $+1 . \mathrm{O}_{q b}$ then joined in the oxidation, leading to fully oxidized silicene by means of the combination of $\mathrm{O}_{q b}$ and one type of $\mathrm{OH}$ configuration. Therefore, the charge state of Si changed from +1 to +4 during the oxidation of silicene in air $^{20}$.

We have shown that a variety of SOs may be produced by the oxidation of silicene. The spectrum of electronic properties associated with these SOs is even wider than that obtained by the oxidation of graphene ${ }^{32}$. On one hand, we have been provided with an opportunity of making vastly different materials such as metals, semimetals, semiconductors and insulators from the single material of silicene only by means of oxidation. On the other hand, excellent control on the oxidation conditions of silicene needs to be exerted to obtain specific SOs with desired electronic properties. For example, one can control the oxidation of silicene in the atmosphere of oxygen gas to produce partially oxidized silicene with $\mathrm{O}_{o b}^{s}$ or $\mathrm{O}_{o b}^{d}$. This effectively opens the bandgap of silicene without introducing any deep energy levels in the bandgap (Figure 3). The resulting semiconductor (the $\mathrm{SO}$ with $\mathrm{O}_{o b}^{s}$ or $\mathrm{O}_{o b}^{d}$ ) with a bandgap of 0.6 or $0.7 \mathrm{eV}$ can very well remedy the zero-bandgap disadvantage of silicene. We should note that this bandgap opening is much more significant than those induced by vertical electric fields ${ }^{16,17}$. By exquisitely tuning the oxidation of silicene with both $\mathrm{O}$ and $\mathrm{OH}$, one may also produce insulators such as the SOs with $\mathrm{O}_{q b}+\mathrm{OH}_{b l}$ and $\mathrm{O}_{q b}+\mathrm{OH}_{a l}$. These insulating SOs can be readily used in silicene-based devices such as field-effect transistors as gate oxides ${ }^{17,33}$.

\section{Methods}

Density functional theory (DFT) as implemented in the Vienna ab initio simulation package (VASP) with the projector-augmented wave (PAW) method $^{34,35}$ has been employed in this work. The Perdew-Burke-Ernzerhof (PBE) correlation exchange functional at the generalized gradient approximation (GGA) level is adopted. The structures of silicene and SOs are initially optimized at $0 \mathrm{~K}$. During the optimization the total energy and atomic forces for each structure are minimized. The sampling of reciprocal space is performed with the Monkhorst-Pack scheme $e^{36}$. The meshes of $(5 \times$ $5 \times 1)$ and $(9 \times 9 \times 1)$ special $\mathrm{k}$ points are used for the structural relaxation and density-of-states (DOS) calculation, respectively. Depending on the symmetry of the unit cell for a specific structure, an appropriate path in the first Brillouin zone is adopted to calculate the band structure ${ }^{37}$. All the calculations proceed until the changes in energy and the force on each atom are less than $1 \times 10^{-6} \mathrm{eV}$ per cell and 1 $\times 10^{-5} \mathrm{eV} / \mathrm{A}$, respectively. The Becke, three-parameter, Lee-Yang-Parr (B3LYP) exchange-correlation functional has also been used to calculate the bandgaps of SOs after their structures are optimized.

1. Feng, B. et al. Evidence of silicene in honeycomb structures of silicon on $\mathrm{Ag}(111)$. Nano Lett. 12, 3507-3511 (2012).

2. Tsai, W. F. et al. Gated silicene as a tunable source of nearly $100 \%$ spin-polarized electrons. Nat. Commun. 4, 1500 (2013).

3. Meng, L. et al. Buckled silicene formation on $\operatorname{Ir}(111)$. Nano Lett. 13, 685-690 (2013).

4. De Padova, P. et al. Multilayer silicene nanoribbons. Nano Lett. 12, 5500-5503 (2012).

5. Lalmi, B. et al. Epitaxial growth of a silicene sheet. Appl. Phys. Lett. 97, 223109 (2010).

6. Kim, U. et al. Synthesis of Si nanosheets by a chemical vapor deposition process and their blue emissions. ACS Nano, 5, 2176-2181 (2011).

7. Okamoto, H., Sugiyama, Y. \& Nakano, H. Synthesis and modification of silicon nanosheets and other silicon nanomaterials. Chem-Eur. J. 17, 9864-9887 (2011). 
8. Fleurence, A. et al. Experimental evidence for epitaxial silicene on diboride thin films. Phys. Rev. Lett. 108, 245501 (2012).

9. Xu, M. S., Liang, T., Shi, M. M. \& Chen, H. Z. Graphene-like two-dimensional materials. Chem. Rev. 113, 3766-3798 (2013).

10. Koski, K. J. \& Cui, Y. The New Skinny in two-dimensional nanomaterials. ACS Nano 7, 3739-3743 (2013).

11. Kara, A. et al. A review on silicene-new candidate for electronics. Surf. Sci. Rep. 67, 1-18 (2012).

12. Chiappe, D., Grazianetti, C., Tallarida, G., Fanciulli, M. \& Molle, A. Local electronic properties of corrugated silicene phases. Adv. Mater. 24, 5088-5093 (2012).

13. Geim, A. K. Graphene: status and prospects. Science 324, 1530-1534 (2009).

14. Liu, C. C., Feng, W. \& Yao, Y. Quantum spin hall effect in silicene and twodimensional germanium. Phys. Rev. Lett. 107, 076802 (2011).

15. Chen, L. et al. Evidence for dirac fermions in a honeycomb lattice based on silicon. Phys. Rev. Lett. 109, 056804 (2012).

16. Drummond, N. D., Zólyomi, V. \& Fal'ko, V. I. Electrically tunable band gap in silicene. Phys. Rev. B 85, 075423 (2012).

17. Ni, Z. et al. Tunable bandgap in silicene and germanene. Nano Lett. 12, 113-118 (2012).

18. Ezawa, M. Valley-polarized metals and quantum anomalous hall effect in silicene. Phys. Rev. Lett. 109, 055502 (2012).

19. De Padova, P., Quaresima, C., Olivieri, B., Perfetti, P. \& Le Lay, G. Strong resistance of silicene nanoribbons towards oxidation. J. Phys. D: Appl. Phys. 44, 312001 (2011).

20. Molle, A. et al. Hindering the oxidation of silicene with non-reactive encapsulation. Adv. Func. Mater. 23, 4340 (2013).

21. Deal, B. E. \& Grove, A. S. General relationship for the thermal oxidation of silicon. J. Appl. Phys. 36, 3770-3778 (1965).

22. McDowell, M. T. et al. Novel size and surface oxide effects in silicon nanowires as lithium battery anodes. Nano Lett., 11, 4018-4025 (2011).

23. Ma, D. D. D. et al. Small-diameter silicon nanowire Surfaces. Science 299, 1874-1877 (2003)

24. Wolkin, M. V., Jorne, J., Fauchet, P. M., Allan, G. \& Delerue, C. Electronic states and luminescence in porous silicon quantum dots: the role of oxygen. Phys. Rev. Lett. 82, 197-200 (1999).

25. Chen, X. B., Pi, X. D. \& Yang, D. Bonding of oxygen at the oxide/nanocrystal interface of oxidized silicon nanocrystals: an ab initio study. J. Phys. Chem. C 114, 8774-8781 (2010).

26. Wang, Y. \& Ding, Y. Mechanical and electronic properties of stoichiometric silicene and germanene oxides from first-principles. Phys. Status. Solid-R 7, 410 (2013).

27. Mattson, C. et al. Evidence of nanocrystalline semiconducting graphene monoxide during thermal reduction of graphene oxide in vacuum. ACS Nano 5 , 9710-9717 (2011).

28. Sivek, J., Sahin, H., Partoens, B. \& Peeters, F. Adsorption and absorption of boron, nitrogen, aluminum, and phosphorus on silicene: stability and electronic and phonon properties. Phys. Rev. B 87, 085444 (2013).
29. Cahangirov, S., Topsakal, M., Aktürk, E., Şahin, H. \& Ciraci, S. Two- and onedimensional honeycomb structures of silicon and germanium. Phys. Rev. Lett. 102, 236804 (2009).

30. Houssa, M., Pourtois, G., Afanas'ev, V. V. \& Stesmans, A. Can silicon behave like graphene? A first-principles study. Appl. Phys. Lett. 97, 112106 (2010).

31. $\mathrm{Li}, \mathrm{Y}$. \& Chen, $\mathrm{Z}$. XH/ $\pi(\mathrm{X}=\mathrm{C}, \mathrm{Si})$ Interactions in graphene and silicene: weak in strength, strong in tuning band structures. J. Phys. Chem. Lett. 4, 269-275 (2013).

32. Yan, J. A., Xian, L. D. \& Chou, M. Y. Structural and electronic properties of oxidized graphene. Phys. Rev. Lett. 103, 086802 (2009).

33. Ezawa, M. Quantized conductance and field-effect topological transistor in silicene ribbons. Appl. Phys. Lett. 102, 172103 (2013).

34. Blöchl, P. E. Projector augmented-wave method. Phys. Rev. B 50, 17953-17979 (1994).

35. Kresse, G. \& Joubert, D. From ultrasoft pseudopotentials to the projector augmented-wave method. Phys. Rev. B 59, 1758-1775 (1999).

36. Monkhorst, H. J. \& Pack, J. D. Special points for Brillouin-zone integrations. Phys. Rev. B, 13, 5188-5192 (1976).

37. Liu, Z. Modulation of Dirac points and band-gaps in graphene via periodic fullerene adsorption. AIP Adv. 3, 052126 (2013).

\section{Acknowledgments}

We are grateful to Prof. Kehui Wu at Institute of Physics, CAS for commenting on our manuscript. This work is mainly supported by the 973 program (Grant No. 2013CB632101) and the NSFC for excellent young researchers (Grant No. 61222404). Partial support is provided by the R\&D Program of Ministry of Education of China (Grant No. 62501040202). Shanghai Supercomputer Center is acknowledged for providing computation resources.

\section{Author contributions}

X.D.P. and R.W. conceived the idea. R.W. performed the DFT calculations and analyzed the data. X.D.P., R.W. and Z.Y.N. discussed the calculation details and results together. Y.L. provided the Vienna ab initio simulation package (VASP) and guided the DFT calculations. S.S.L. and M.S.X. contributed to the data analysis. R.W., X.D.P. and D.R.Y. wrote the manuscript.

\section{Additional information}

Supplementary information accompanies this paper at http://www.nature.com/ scientificreports

Competing financial interests: The authors declare no competing financial interests. How to cite this article: Wang, R. et al. Silicene oxides: formation, structures and electronic properties. Sci. Rep. 3, 3507; DOI:10.1038/srep03507 (2013).

\footnotetext{
(c) (i) $\odot$ This work is licensed under a Creative Commons AttributionNonCommercial-NoDerivs 3.0 Unported license. To view a copy of this license, visit http://creativecommons.org/licenses/by-nc-nd/3.0
} 